\title{
Semi-quantitative risk assessment by expert elicitation of potential introduction routes of African swine fever from wild reservoir to domestic pig industry and subsequent spread during the Belgian outbreak (2018-2019)
}

\author{
Axel Mauroy $^{1}$ (D) | Pieter Depoorter ${ }^{1}$ | Claude Saegerman $^{2 *}$ (D) | Brigitte Cay ${ }^{3}$ | Nick De \\ Regge $^{3} \mid$ Maria-Eleni Filippitzi ${ }^{4}$ | Claude Fischer $^{5}$ | Martine Laitat ${ }^{6}$ | Dominiek Maes ${ }^{7}$ (D) \\ Kevin Morelle ${ }^{8,9}$ (i) | Hans Nauwynck ${ }^{10}$ | Xavier Simons ${ }^{4}$ | Thierry van den Berg ${ }^{11}$ | \\ Xavier Van Huffel $^{1}$ | Etienne Thiry ${ }^{12}$ (I) | Jeroen Dewulf $7^{7}$ (I) \\ ${ }^{1}$ Staff Direction for Risk Assessment, Directorate General Control Policy, Federal Agency for the Safety of the Food Chain, Bruxelles, Belgium \\ ${ }^{2}$ Faculty of Veterinary Medicine, Research Unit in Epidemiology and Risk Analysis Applied to Veterinary Sciences (UREAR-ULiège), Fundamental and Applied \\ Research for Animal Health (FARAH) Centre, University of Liège, Liège, Belgium \\ ${ }^{3}$ Service of Enzootic, Vector-Borne and Bee Diseases, Scientific Direction Infectious Diseases in Animals, Sciensano, Brussels, Belgium \\ ${ }^{4}$ Veterinary Epidemiology Unit, Department of Epidemiology and Public Health, Sciensano, Brussels, Belgium \\ ${ }^{5}$ Dept. Nature Management, University of Applied Sciences of Western Switzerland, Geneva, Switzerland \\ ${ }^{6}$ Faculty of Veterinary Medicine, Swine Clinic, Clinical Department of Production Animals, University of Liège, Liège, Belgium \\ ${ }^{7}$ Faculty of Veterinary Medicine, Department of Reproduction, Obstetrics and Herd Health, Ghent University, Merelbeke, Belgium \\ ${ }^{8}$ Faculty of Forestry and Wood Sciences, Department of Game Management and Wildlife Biology, Czech University of Life Sciences, Prague, Czech Republic \\ ${ }^{9}$ Department of Migration, Max Planck Institute of Animal Behavior, Radolfzell, Germany \\ ${ }^{10}$ Faculty of Veterinary Medicine, Department of Virology, Parasitology and Immunology, Ghent University, Merelbeke, Belgium \\ ${ }^{11}$ Scientific Directorate Animal Infectious Diseases, Sciensano, Brussels, Belgium \\ ${ }^{12}$ Faculty of Veterinary Medicine, Veterinary Virology, FARAH Centre, University of Liège, Liège, Belgium
}

\section{Correspondence}

Axel Mauroy, Staff direction for Risk Assessment, Directorate general Control Policy, Federal Agency for the Safety of the Food Chain, CA Botanique Blvd. du Jardin Botanique 55, 1000 Bruxelles, Belgium. Email: axel.mauroy@favv-afsca.be

\begin{abstract}
Since the introduction in Georgia in 2007 of an African swine fever (ASF) genotype 2 virus strain, the virus has rapidly spread to both Western European and Asian countries. It now constitutes a major threat for the global swine industry. The ongoing European transmission cycle has been related to the 'wild boar habitat' with closed transmission events between wild boar populations and incidental spillovers to commercial and non-commercial (backyard) pig holdings. During the epidemic in Belgium, only wild boar were infected and although the introduction route has not yet been elucidated, the 'human factor' is highly suspected. While ASF was successfully contained in a small region in the Southern part of Belgium without affecting domestic pigs, the risk of spillover at the wild/domestic interface remains poorly assessed. In this study, we used a semi-quantitative method, involving national and international experts, to assess the risk associated with different transmission routes for ASF introduction from wild boar to domestic pig holdings and subsequent dissemination
\end{abstract}


between holdings in the Belgian epidemiological context. Qualitative responses obtained by our questionnaire were numerically transformed and statistically processed to provide a semi-quantitative assessment of the occurrence of the hazard and a ranking of all transmission routes. 'Farmer', 'bedding material', 'veterinarian' and 'professionals from the pig sector' were considered as the most important transmission routes for ASF introduction from the wild reservoir to pig holdings. 'Animal movements', 'farmer', 'veterinarian', 'iatrogenic', 'animal transport truck' and 'animal care equipment' were considered as the most important transmission routes posing a risk of ASF spread between pig holdings. Combined with specific biosecurity checks in the holdings, this assessment helps in prioritizing risk mitigation measures against ASF introduction and further spread in the domestic pig industry, particularly while the ASF situation in Western Europe is worsening.

KEYWORDS

African swine fever, pig, risk assessment, spread, wild boar

\section{1 | INTRODUCTION}

African swine fever (ASF) is a viral disease (caused by ASF virus of the family Asfarviridae) notifiable to the EU and the OIE (Alonso et al., 2018; OIE, 2019). ASF only affects Suidae, in particular domestic pigs (Sus scrofa domesticus) and wild boar (Sus scrofa). In 2007, ASF was introduced to Georgia, probably from the Eastern part of Africa or Madagascar via contaminated food used to feed pigs (Blome et al., 2020) (EFSA, 2020). An early isolate, Georgia 2007/01, was genetically related to the genotype II of ASF viruses (Rowlands et al., 2008). It then spread quickly to neighbouring countries, from Russia to the Baltic countries (Latvia, Lithuania, Estonia) and Poland, and further towards Western Europe. Currently, many Eastern European countries have reported high numbers of infected domestic pigs and/or wild boar, both as single outbreak or multiple localized outbreaks (Belarus, Moldavia, Ukraine, Poland, Czech Republic, Hungary, Slovakia, Romania, Bulgaria, Greece, Serbia) (EFSA, 2020). The virus has been recently detected in Western Europe as well, in Belgium in 2018 (Garigliany et al., 2019; Gilliaux et al., 2019; Linden et al., 2019; Saegerman et al., 2018) and more recently in 2020 in Germany at the border with Poland (OIE, 2020; Sauter-Louis et al., 2020)). Simultaneously, the virus spread eastwards through Russia to reach mainland China and now numerous other Asian countries. Thereby, ASF has become a major global threat for the swine industry.

The ASF virus infects its host mainly through the oronasal route and is very resistant in the environment, especially when associated with biological material (blood, meat, cadavers, faeces) (Blome et al., 2020; Guinat et al., 2016; Sánchez-Vizcaíno et al., 2012). Many potential transmission routes allow the virus to be transmitted from an infected animal to a healthy one. They are of both direct (via infected animals, their excretions and their cadavers) and indirect nature (via animate or non-animate vectors such as mechanical carriage of the virus by humans, contaminated clothing or equipment) (Costard, Mur et al., 2013; Guinat et al., 2016; SánchezVizcaíno et al., 2012). Soft ticks (Ornithodoros porcinus moubata and Ornithodoros marocanus) are involved in transmission cycles in Africa and have been involved in an outbreak in the Iberian peninsula (Portugal and Spain) in the 1960's (Sánchez-Vizcaíno et al., 2015). More recently, haematophagous flies have been also proposed as non-multiplying vectors for mechanical transmission of the ASF virus between porcine hosts (Olesen, Hansen et al., 2018; Olesen, Lohse et al., 2018; Fila \& Woźniakowski, 2020; Olesen et al., 2020; Saegerman et al., 2020; Vergne et al., 2020).

The spread of ASF in wildlife (wild boar) has shown different transmission patterns across Europe. The most frequent pattern is associated with transmission events between wild boar populations. These lead sometimes to spillover events to domestic pigs, usually held 'outdoor' and on 'non-commercial' farms, also known as 'backyard' farms (Jurado et al., 2018). The sustained circulation of the ASF virus within wild boar population is a fully novel 'wild boar-habitat' transmission cycle where direct or indirect contacts (through cadavers of infected wild boars or leftovers from it) can sustain the infections (Chenais et al., 2018). Occasionally, ASF 'makes a jump' from an endemic area to a more distant area, that is ASF suddenly appears in new areas far away from endemic zones for which the spread cannot be explained by wild boar contacts. These introduction events have been suspected to be related to human mediated spread (the so-called 'human factor') through the import of infectious animals or material in naïve areas (EFSA, 2020). At least three representative examples of such suspected human-mediated introductions can be listed: introduction into wildlife in the Czech Republic in 2017, in wildlife in Belgium in 2018 and more recently in 2020 in wildlife in Western Poland (EFSA, 2020) (OIE, 2020).

In 2014, Roelandt and collaborators assessed the overall risk of ASF entry in Belgium as 'low' (Roelandt et al., 2017). This was 
based on the probability of introduction estimated to be 'low' and the consequences estimated to be high'. They pointed out the potential introduction risks (and uncertainties) through illegal importation of local meat products through the eastern European seasonal workforce in Belgium, through hunting tourism and through wild boar. In September 2018, wild boar from a small area in the south of Belgium were confirmed as infected by ASF (Linden et al., 2019). Huge efforts (i.e. regionalization, hunt, traps, fences, search and removal of cadavers) have been carried out by the Regional authorities for containment and eradication of the outbreak. At the same time, Belgian federal authorities (Federal Agency for the Safety of the Food Chain, FASFC) increased the level of awareness and vigilance in the domestic pig sector to early detect any spillover event from the wild reservoir. In early September 2018 after the first cases, two zones, zone I (or buffer zone) and zone II (or contaminated zone), were defined according to the European regulation concerning ASF. A temporary stand still was imposed on the entire Belgian pig industry and a stamping out of pig holdings localized within the contaminated zone (where infected wild boar were present) was quickly carried out. As this zone was later further extended following the discovery of infected wild boar outside, preventive culling was no longer carried out systematically but replaced by a systematic biosecurity assessment of the newly included farms by the FASFC. Up to late August 2019, infected live wild boar and cadavers at various stages of decomposition were removed from the contaminated zone and tested. Since August 2019, only bones giving positive result by $\mathrm{PCR}$ (but without residual infectivity in cell culture assay - B. Cay (personal communication) have been discovered, the last one being discovered in March 2020. Presently, Belgium has recovered its status as 'officially free from ASF' according to the OIE's conditions.

The Scientific Committee established at the FASFC delivered from October 2018 to June 2020 ten opinions to scientifically support Belgian authorities in their efforts to manage the disease. One of these opinions (SciCom, 2020) aimed to provide the risk managers with a semi-quantitative assessment of the putative risk transmission routes for introduction of ASF from wildlife to the domestic pig sector in the particular situation of Belgium. This opinion was based on statistical analysis of the responses delivered by 34 national and international experts in veterinary virology, veterinary epidemiology, domestic pig industry, wildlife and wildlife disease management, to a survey on transmission routes for ASF introduction from the wild reservoir to the domestic pig sector giving the particular conditions of the Belgian epidemiological context.

\section{2 | MATERIAL AND METHODS}

The World Organisation for Animal Health (OIE) defines a risk as the likelihood of the occurrence and the likely magnitude of the biological and economic consequences of an adverse event or effect to animal or human health (Dufour et al., 2011). In this study, biological and economic consequences for occurrence of the hazard (ASF introduction in the Belgian pig industry) were not considered as they are well known and particularly heavy (direct losses plus indirect impact linked to the loss of the 'officially free from ASF in Suidae' status for the country and all economic consequences on trades). These severe consequences are unavoidably increasing the level of the final qualitative risk which can be assessed from any transmission routes or risk pathways of any hazard, even if its probability of occurrence can be assessed as relatively low. Instead, a list of various transmissions routes for ASF introduction at the wild/domestic interface and those for further spread between pig holdings was provided to an expert panel, in order to assess the likelihood of occurrence of the risk. Individual scores for occurrence of the hazard were thereof obtained.

\section{1 | Putative transmission routes}

Twenty-five transmission routes that could lead to ASF introduction from wildlife into a pig holding were identified by expert opinion ( $n=15$ experts; all co-authors except X. Van Huffel) and based on literature data (Bellini et al., 2021; Costard, Mur et al., 2013; Guinat et al., 2016; Sánchez-Vizcaíno et al., 2012). The following transmission routes were considered for the risk of ASF introduction into a pig holding:

- those related to infected wild boar living in an area of the country ( $n=2$ routes): infected living wild boar ('living wild boar'), infected wild boar cadaver/trophies from an infected wild boar ('wild boar cadaver/trophies, the later relates to some pieces from the wild boar that hunters can bring back at home');

- those which are human-related ( $n=8$ routes), if these people were involved with activities within the regulated zone: 'farmer' (the operator in a pig holding), 'veterinarian' (any practitioner involved in a pig holding), 'hunter', 'fisherman', 'hiker' (people walking around in the contaminated zone where infected wild boar reside, 'crop/forest worker' (people working inside the contaminated zone), 'professional from the pig sector', 'non-professional visitor' (of a pig holding who has been staying in a contaminated area);

- those related to material for animal care $(n=2)$ : 'farm vehicle/ feeding equipment', 'bedding material';

- those related to 'arthropods' ( $n=1)$ i.e. ticks, haematophagous or other insects and flies, maggots;

- those related to animals other than wild boar ( $n=8)$ : 'rodents', 'hunting dog', 'farm dog', 'ruminant', 'horse', 'bird', 'cat', 'wild animals' (other than wild boar);

- those related to feed for pigs ( $n=2)$ : 'swill feeding', 'contaminated vegetal products' (as used as feed, i.e. cereals, grass, soy);

- those related to water $(n=2)$ : 'well water', 'river water'.

Twenty-five transmission routes that could facilitate the further spread of ASF between pig holdings following any introduction 
event were identified by the same expert group. The following transmission routes were considered for the risk of ASF spread between pig holdings (EFSA, 2014):

- those related to an infected pig $(n=5)$ : 'animal movements', 'airborne', 'animal by-products' (in particular manure), 'blood products' of swine origin, 'embryo/sperm/oocysts';

- those which are human-related $(n=5)$, when people are involved in activities within the holding: 'farmer', 'veterinarian' (any practitioner involved in pig holding), 'controller for authority' (federal and regional agents in charge for the controls carried out in holdings for compliance with regulations), 'professional from the pig sector' (who can be in close contacts with pigs as animal trader, butcher, slaughter employee, feed trader, holding employee, etc.), 'non-professional visitor' (of the holding);

- those which are vehicle-related ( $n=5)$ : 'feed truck', 'animal transport truck', 'rendering truck', 'manure transport truck', 'inseminator's vehicle' (i.e. boar semen delivery vehicle);

- those related to material ( $n=4)$ : 'iatrogenic' (syringe, needles), 'animal care equipment', 'feeding equipment', 'bedding material';

- those which are pest-related $(n=2)$ : 'haematophagous arthropods' (flies, ticks, other), 'rodents';

- those which are animal-related ( $n=4)$ (other than pigs and if in contact with the ASF virus in the infected holding): 'dog', 'cat', 'ruminant', 'bird'.

\subsection{Questionnaire and elicited experts}

A questionnaire, listing the transmission routes to be assessed by the experts was developed in excel (Data S1). The questionnaire was prepared and validated by all the co-authors of this study (all members of the working group of the Scientific Committee established at the FASFC on ASF) in order to fulfil usual consideration in expert elicitation (details on the assessment, description of the Belgian context for ASF, use of the form and scoring scheme explanation) before sending. This was send by mail to a group of European experts $(n=77)$ together with the instructions on how to fill the questionnaire. Both national and international experts were identified by the authors They were proposed based upon their expertise on epidemiology and/or virology of ASF and other infectious pig diseases as well as expertise on wildlife and wildlife management. All the co-authors except the first author of this study also filled in the questionnaire. Data S2 gives the name, origin and field of expertise of each expert. Each expert was asked to qualitatively assess each transmission route on the following three criteria: (a) the likelihood of contamination by ASF, (b) the likelihood of transmission of ASF to domestic pigs, (c) the frequency of contact between domestic pigs and the transmission route. Every criterion had to be ranked on a 6rank scale (range: 'negligible', 'very low', 'low', 'medium', 'high', 'very high'). Each qualitative value was further explained in the questionnaire in order to help to the standardization and the assessors to perceive this 6-rank scale. Furthermore, the experts were asked to assess the level of uncertainty associated to each response (range: 'low', 'medium', 'high') as well as their level of expertise (range: 'negligible', 'very low', 'low', 'medium', 'high', 'very high') in their respective fields (e.g. veterinary virology, veterinary epidemiology, pig industry, wildlife disease and management), and their knowledge on the particular Belgian ASF epidemiological context (range: 'negligible', 'very low', 'low', 'medium', 'high', 'very high'). It was clearly specified that the assessment must be filled in keeping in mind the particular epidemiological context of Belgium for ASF (that was further detailed in the form). It was assumed and specified to the experts that all the transmission routes for the virus must be considered as infected/ contaminated and/or originating or having circulated in the contaminated zone delimitated in Belgium in compliance with the regionalization within the European regulation (zones I and II). Regarding all transmission routes for ASF spreading, it was specified that they must be assessed before any primary detection of virus circulation, i.e. before any mitigation measure or actions are put in force.

\section{3 | Statistics}

All statistical analyses were carried out using $\mathrm{R}$ and Statistica. In order to perform the analyses, the qualitative responses were first transformed into numerical values, that is 'negligible' $=0$, 'very low' $=1$, 'low' $=2$, 'medium' $=3$, 'high' $=4$, 'very high' $=5$ for each criteria and 'low' $=1$, 'medium' $=2$, high' $=3$ for uncertainty on. The numerical value ' 0 ' was given to the qualitative assessment 'negligible' as it was reflecting a level considered as sufficiently low to be ignored and allowing numerical transformation relying on equidistance between each of the nominal value (Likert scale). For each transmission route, an overall score for occurrence of the hazard was calculated by multiplying the individual scores for each of the three corresponding criteria's (i.e. likelihood of contamination by ASF, likelihood of transmission of ASF to domestic pigs, frequency of contact between domestic pigs and the transmission route). Finally, the different transmission routes were ranked for both ASF introduction and ASF spread, based on their median overall scores. Furthermore, an overall score of uncertainty around the responses was included, by adding individual scores for uncertainty on each assessed criterion and calculating the median thereof.

\subsection{Sensitivity analysis}

A sensitivity analysis was performed according to the same methodology developed by Saegerman et al., (2020) to analyse the robustness of the expert elicitation and the putative influence of each criteria in the ranking of transmission routes for both introduction and spread of ASF. Briefly, a ranking of transmission routes, considering all criteria, that is the probability of contamination by ASF, the probability of transmission of ASF to domestic pigs as well as the frequency of contact, was first established as reference and using the median value of the overall score. One 

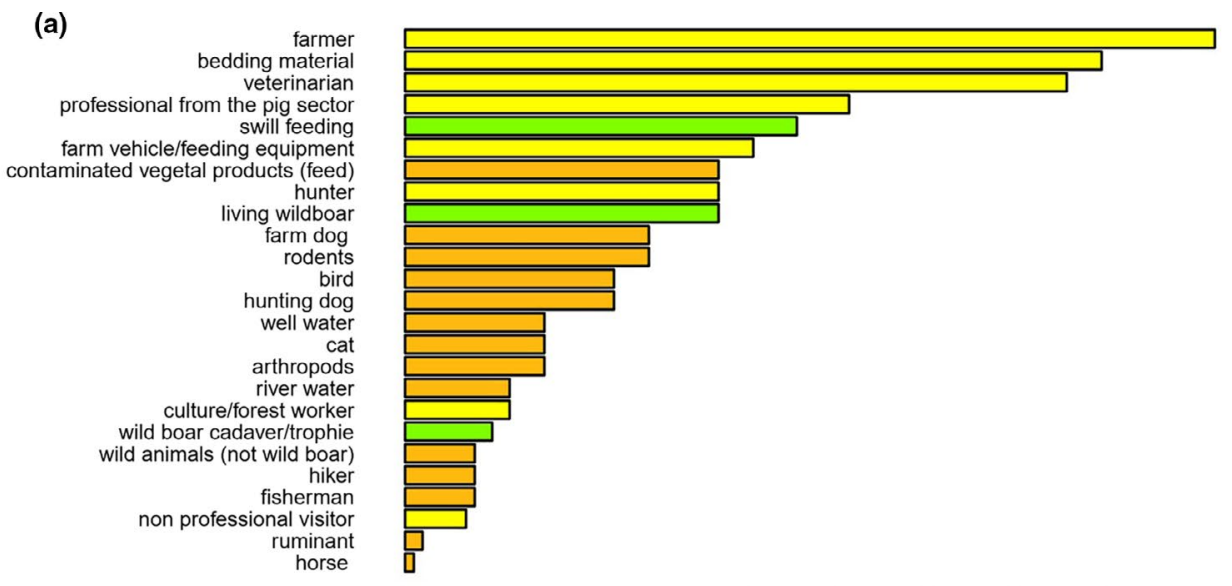

bing

vell water

cat
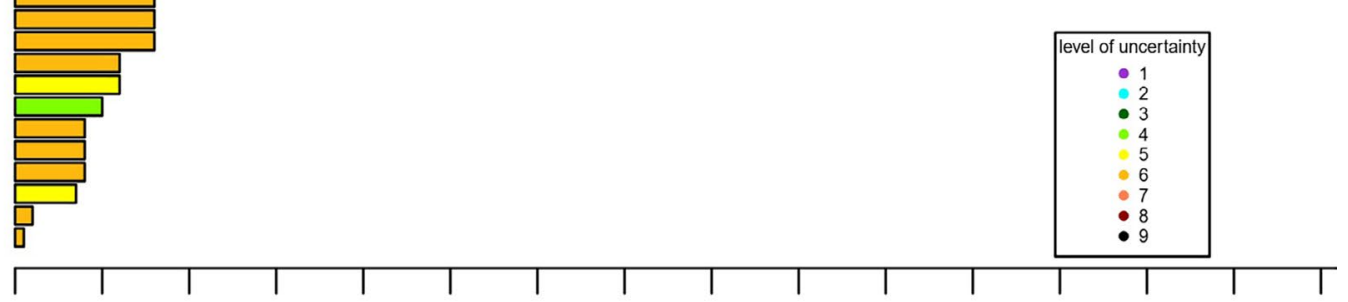
5

10

15

$20 \quad 25$

30

35

40

45

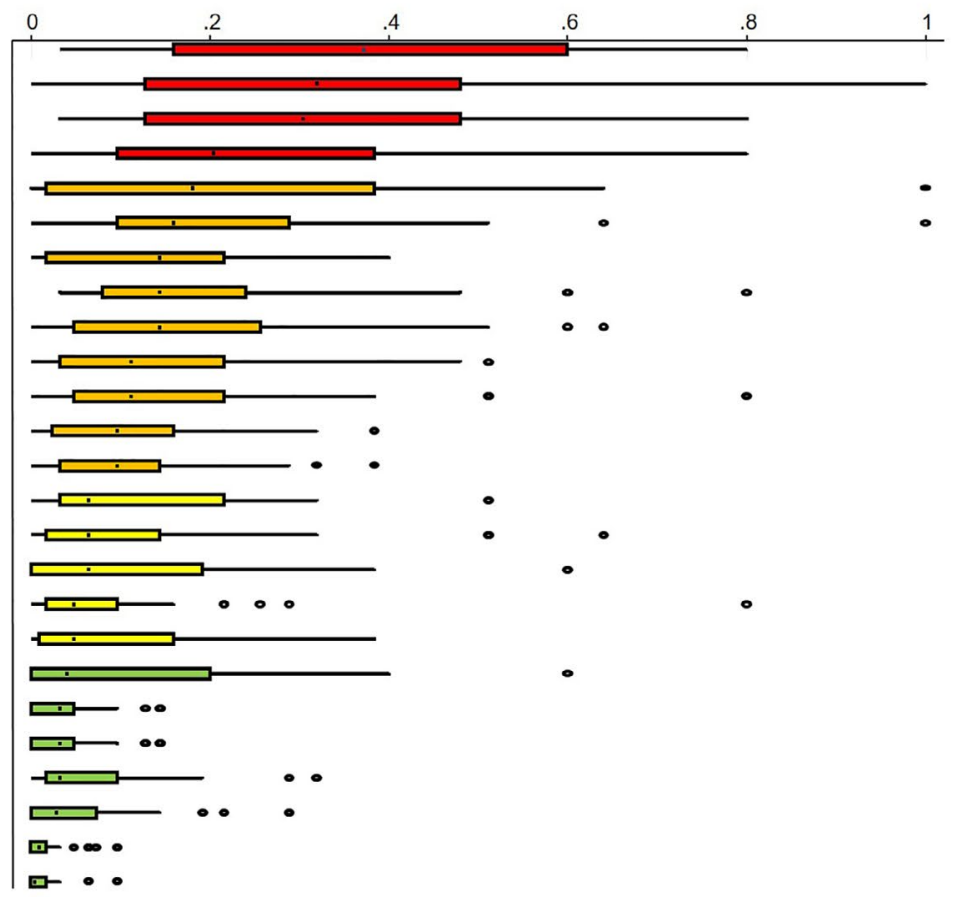

1

01 animal farmer $\left({ }^{* *}\right)$

02 bedding material $\left(^{\star \star}\right)$

03 veterinarian $(* *)$

04 professionally closely involved with pigs ${ }^{(*)}$

05 swill feeding $\left.{ }^{* *}\right)$

06 farm vehicle and feeding equipment $\left.{ }^{* *}\right)$

07 living wild boar $\left.{ }^{* *}\right)$

08 hunter $\left({ }^{*}\right)$

09 contaminated vegetal products $\left(^{\star \star *}\right)$

10 rodents $\left({ }^{\star \star *}\right)$

11 farm $\operatorname{dog}\left({ }^{\star \star \star}\right)$

12 hunting $\operatorname{dog}(* * *)$

13 bird $\left({ }^{* * *}\right)$

14 arthropods $\left(^{\star \star \star}\right)$

15 cat $\left.{ }^{* * *}\right)$

16 well water $\left(^{\star \star \star}\right)$

17 persons involved with cultures and forests $\left(^{* *}\right)$

18 river water $\left(^{* \star *}\right)$

19 wild boar cadaver or trophy $\left({ }^{\star *}\right)$

20 fisherman $\left.{ }^{(* *}\right)$

21 hiker $\left.{ }^{* * *}\right)$

22 wild animals other than wild boar $\left({ }^{* * *}\right)$

23 non professional visitor $\left.{ }^{(* *}\right)$

24 ruminant $\left({ }^{* * *}\right)$

25 horse $\left(^{\star \star \star}\right)$

Group 1

Mean $\$=0.300$

S.D. $=0.061$

$(\mathrm{N}=4)$

Group 2

Mean $=\mathbf{0 . 1 3 2}$

S.D. $=0.028$

$(N=9)$

Group 3

Mean $=0.058$

S.D. $=0.008$

$(\mathrm{N}=5)$

Group 4

Mean $=0.025$

S.D. $=0.013$

$(\mathrm{N}=7)$

FIGURE 1 a. Ranking on decreasing median overall scores of the 25 transmission routes identified for the risk of ASF introduction into pig holdings. Median overall score is the median obtained on overall scores as assessed by 34 experts(overall score for each transmission route and for each expert was obtained by multiplication of the individual scores on three criteria, that is the likelihood of contamination by ASF, the likelihood of transmission of ASF to domestic pigs and the frequency of contact between domestic pigs and the transmission route). The median level of uncertainty is given following a colour code. Median level of uncertainty was obtained in a similar way than for the median overall score but by addition of the individual score of uncertainty on each criterion. b. Risk groups for the introduction of African swine fever into pig holdings, obtained following regression tree analysis. The groups range from 1 to 4 , from the group with the highest score for occurrence of hazard (group 1) to the lowest risk (group 4). The overall scores for occurrence of hazard were transformed to risk scores, and the transmission routes were ranked according to their median score (dot within the box) for occurrence of hazard reported as a proportion ( 0 to 1 on $\mathrm{X}$-axis). The median score of uncertainty was scaled from 1 to 3 and represented in the figure with asterisks: low $\left({ }^{*}\right)$, medium $\left({ }^{* *}\right)$, high $\left(^{* * *}\right)$ uncertainty. \$: median value of risk score

criterion (one by one) was then ignored, and all transmission routes were subsequently ranked using the same methodology as presented before. For each transmission route, modifications in the ranking were counted, only considering changes of more than three ranks. A diagram was created to visualize any modification of rank induced by the withdrawal of a given criterion of transmission routes. In the same way, the robustness of expert elicitation was tested using the same methodology (removal of all national or international experts, and by the removal of 14 experts randomly selected permitting the artificial constitution of 10 panels of 20 


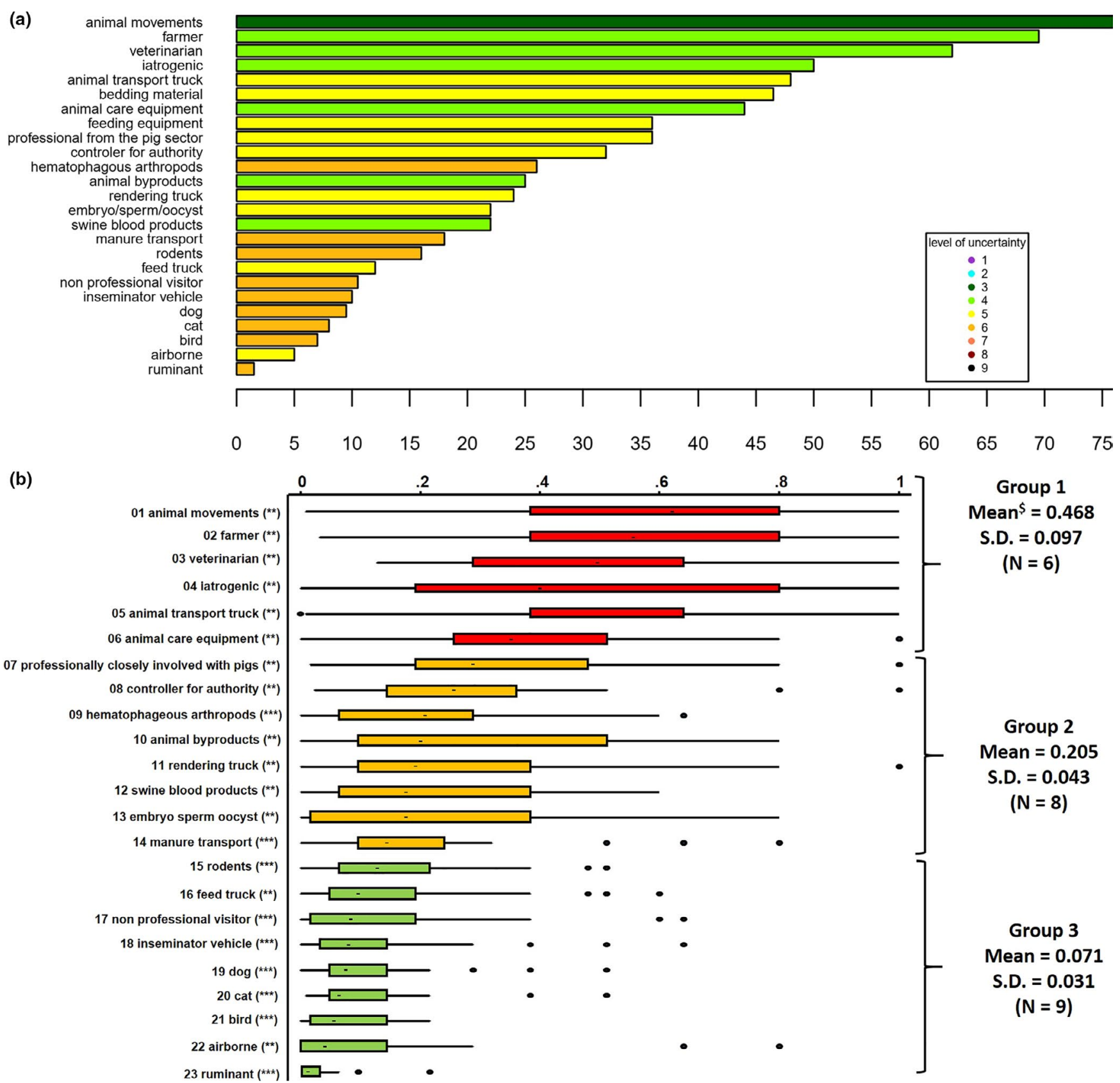

FIGURE 2 a. Ranking on decreasing median overall scores of the 23 transmission routes identified for the risk of ASF spread between pig holdings. Median overall score is the median obtained on overall scores as assessed by 34 experts (overall score for each transmission route and for each expert was obtained by the multiplication of the individual scores on three criteria, that is the likelihood of contamination by ASF, the likelihood of transmission of ASF to domestic pigs, the frequency of contact between domestic pigs and the transmission route). The median level of uncertainty is given following a colour code. Median level of uncertainty was obtained in a similar way than for the median overall score but by addition of the individual score of uncertainty on each criterion. b. Risk groups for the spread of African swine fever between pig holdings, obtained following regression tree analysis. The groups range from 1 to 3 , from the group with the highest score for occurrence of hazard (group 1) to the lowest risk (group 3). The overall scores for occurrence of hazard were transformed to risk scores, and the transmission routes were ranked according to their median score (dot within the box) for occurrence of hazard reported as a proportion (0-1 on X-axis). The median score of uncertainty was scaled from 1 to 3 and represented in the figure with asterisks: low $\left({ }^{*}\right)$, medium $\left({ }^{* *}\right)$, high $\left(^{* * *}\right)$ uncertainty. \$: median value of risk score

experts in order to test the influence of the composition of expert field expertise). Further sensitivity analyses were carried out by making the transmission routes depending on the origin of the experts (national or international) and on their level of expertise in the different field of expertise.

\section{5 | Regression tree analysis}

The objective of the regression tree analysis was to attribute each transmission route of ASF into a homogenous group, that is each group of routes of ASF transmission is statistically different from 
another group. For this regression tree analysis each overall score for each transmission route was transformed in proportions (overall score divided by the maximal score to obtain the 'risk score'). Transmission routes of ASF were grouped in a terminal node, which was characterized by an average overall score with the smallest standard deviation as possible. The regression tree analysis was carried out using Salford Predictive Modeller (Salford Systems, San Diego, USA) (for further details, see Saegerman et al., 2020).

\section{3 | Results}

Thirty-four responses were received ( $n=20$ for national expert; $n=14$ for international experts; response rate of about $44 \%$; details of the respondents are given in Data S2). The level of expertise reached by the panel in the different relevant fields of expertise (virology, epidemiology, pig production sector, wildlife diseases and management, knowledge of the Belgian epidemiological context for ASF) is given in Data S3. Their median level of expertise in epidemiology, pig production sector and knowledge of the Belgian epidemiological context for ASF was self-assessed as 'high' by the expert panel. Their median level of expertise in virology and wildlife diseases and management were self-assessed as 'moderate' and 'low to moderate', respectively. Data S4 summarized the results for as both barplots and boxplots which were inferred from all the assessment for each transmission route (ASF introduction into a pig holding or spread between pig holdings).

The ranking of the transmission routes which was inferred from the assessments is given in Figure 1a. By means of regression tree analysis, four groups could be distinguished according to their relevance for ASF introduction into a pig holding from in the Belgian wild reservoir in the regulated zone (Figure 1b), as to know: (a) group 1 (risk score of $0.3 \pm 0.061$ ): 'farmer', 'bedding material', 'veterinarian' and 'professionals from the pig sector'; (b) group 2 (risk score of $0.132 \pm 0.028$ ): 'swill feeding', 'farm vehicles/feeding equipment', 'contaminated vegetal products', 'hunter', 'living wild boar', 'farm dog', 'rodents' and 'bird'; (c) group 3 (risk score of $0.058 \pm 0.008$ ): 'hunting dog', 'well water', 'cat', 'haematophagous arthropods' and 'river water'; (d) group 4 (risk score of $0.025 \pm 0.013$ ): 'culture/forest worker', 'wild boar cadaver/trophies', 'wild animal other than wild boar', 'hiker', fisherman', 'non-professional visitor', 'ruminant' and 'horse'.

A ranking was also inferred in regard to ASF spread between pig holdings following any introduction (Figure 2a). Regression tree analysis has identified three groups according to their relevance for ASF spread between pig holdings (Figure 2b) (a) group 1 (risk score of $0.468 \pm 0.097$ ): 'animal movements, 'farmer', 'veterinarian', 'iatrogenic', 'animal transport truck' and 'animal care equipment'; (b) group 2 (risk score of $0.205 \pm 0.043$ ): 'professional from the pig sector', 'controller for authority', 'haematophagous arthropods', 'animal by-products', 'rendering truck', 'embryo/sperm/oocysts', 'swine blood products' and 'manure transport'; (c) group 3 (risk score of $0.071 \pm 0.031$ ): 'rodents', 'feed truck', 'non-professional visitor', 'inseminator vehicle' (boar semen delivery vehicle), 'dog', 'cat', 'bird', 'airborne' and 'ruminant'.

Although the transmission routes 'feeding equipment' and 'bedding material' were submitted for expert assessment as they are usually considered as putative transmission route in the literature, these were subsequently excluded from the analysis of the results. The reason was that lending 'feeding equipment' is a very uncommon practice in the majority of commercial Belgian pig holdings. Similarly, 'bedding material' is no longer available for other holdings after use. Instead, the contaminated litter was assessed via the 'manure transport' and animal by-products (manure) transmission routes.

The sensitivity analysis on the criteria for ASF transmission routes of introduction and spread indicated that all the three criteria are useful as the removal of each one influenced the ranking of routes of ASF transmission and especially for the ASF introduction (important changes in the top five ranked routes) (Figure 3 and Data S5). In addition, the sensitivity analysis on experts permitted to assess the robustness of this elicitation (Figure $4 a$ and b, and Data S6). Also, for ASF introduction, there is an important influence of the panel choice (field of expertise; Data S7) but not in extremities of the ranking. However, little influence was observed in the ranking of ASF spread routes as well as after removing all national or international experts. This influence had also less effect, which underlines the need to have a sufficient number of experts with varying expertise in the panel (Figure $4 c$ and $d$ ).

\section{DISCUSSION}

ASF endemicity in several European wild boar populations is a real threat for the pig industry due to the risk of spillover to commercial or non-commercial (backyard) holdings. In the environment, ASF can remain infectious for a long time in wild boar cadavers or leftovers. Some cases of spillover to domestic pigs have been suspected to be related to this virus persistence (EFSA, 2014). Various transmission routes must be considered for ASF introduction at the wild-domestic pig interface, involving multiple risk pathways. In this study, a semi-quantitative assessment and a ranking of both the risk of introduction and further spread in the Belgian epidemiological context is provided. To correctly consider these rankings, sensitivity analyses were carried out in order to discriminate between the effect of the expertise level of the elicited experts in five relevant fields of expertise, as well as on their origin (national versus international). Moreover, this assessment took into account the level of uncertainty on the responses. By splitting the semi-quantitative assessment of the transmission routes on its three criteria (likelihood of contamination, likelihood of transmission and frequency of contacts with pigs), this study is providing useful information for the development of tailor-made mitigation strategies in countries facing ASF circulation in the wildlife reservoir and coping with the risk of ASF introduction and spread to their domestic pig sector.

Different studies looked at the transmission routes for ASF introduction in the pig industry and/or spread between pig holdings. 


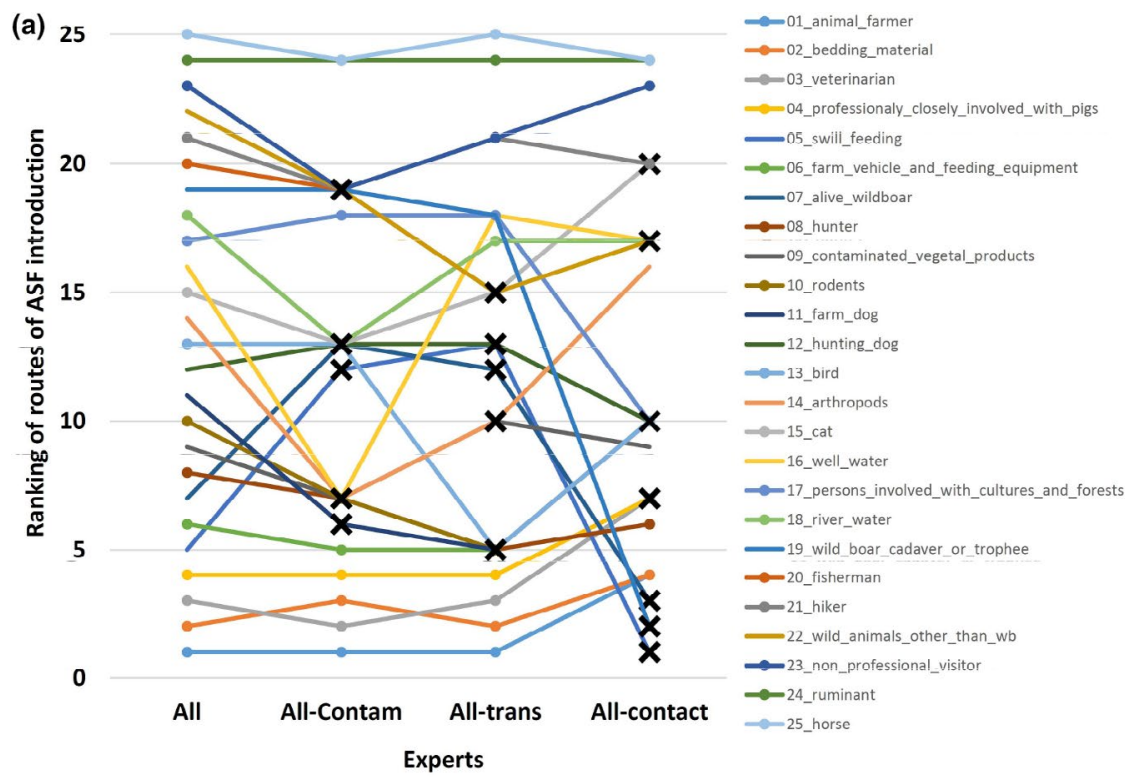

(b)

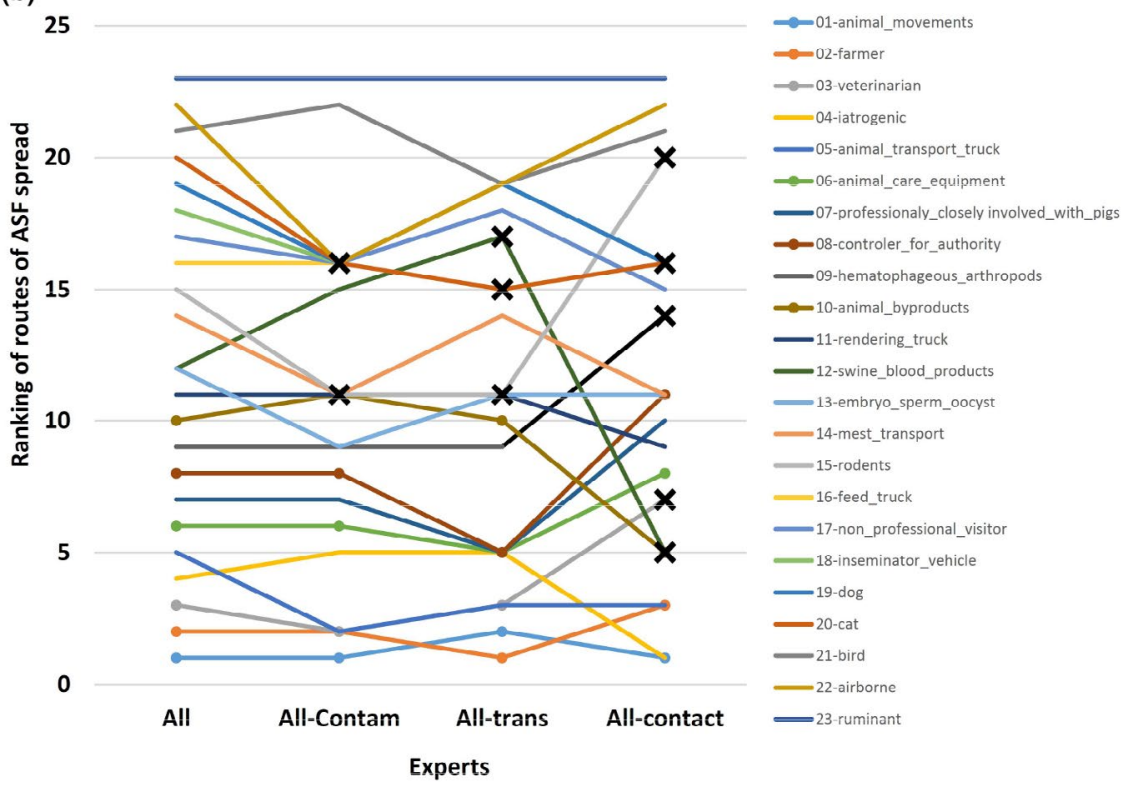

FIGURE 3 Modification in the rank of routes of ASF transmission via sensitivity analysis on criteria, both for introduction [a] and spread [b]. All: all criteria; All-contam: All criteria minus likelihood of contamination by ASF; Alltrans: all criteria minus the likelihood of transmission of ASF to domestic pigs; Allcontact: all criteria minus the frequency of contact with domestic pigs; $\mathrm{X}$ : more than three ranks of difference in the ranking between the particular situation and the situation where all criteria were taken into account
Roelandt and collaborators provide an interesting study about possibilities for ASF introduction in Belgium in 2014, that is 4 years before its introduction in Belgian wild boar (Roelandt et al., 2017). In their study, entry was considered as a whole and all the putative transmission routes were not spread for particular assessment. However, elicited experts did point out potential introduction risks through the local wildlife (Roelandt et al., 2017). At the very beginning of the ASF outbreak in Eastern Europe, Mur and collaborators provide a quantitative risk assessment of ASF entry in European countries through legal import of pigs (Mur et al., 2012). Many similar studies assessed a direct introduction of ASF infected pigs into the local pig industry (Costard, Jones et al., 2013; Mur et al., 2012), rarely looking at intermediary stages in a wildlife reservoir (Cho et al., 2020; Costard, Jones et al., 2013; Herrera-lbatá et al., 2017; Ito et al., 2020; Mur et al., 2012; Sugiura \& Haga, 2018). Yet, considering the current European outbreak, a recent study has demonstrated that ASF cases in domestic pigs and wild boars were spatially correlated in the north west areas of Russia (Vergne et al., 2017). In the specific context of the Belgian outbreak, ASF was already introduced and spread to a susceptible wildlife reservoir which was spatially segregated from the main area of the pig industry. Up to date, only two countries successfully coped with a similar situation during the European outbreak and recovered their status as 'officially free from ASF' according to the OIE's conditions without experiencing any introduction into their domestic pig sector, namely Czech Republic and Belgium.

By means of an expert elicitation, we highlighted four important groups of transmission routes in the risk of ASF transmission from wild boar to pig holdings. The most important routes (group 1) were farmers, bedding material, veterinary practitioners and professionals in the pig sector. The assessment of these four routes showed a lower uncertainty compared with the assessment of most of the other routes, which further reinforces their relative importance. This 
(a)

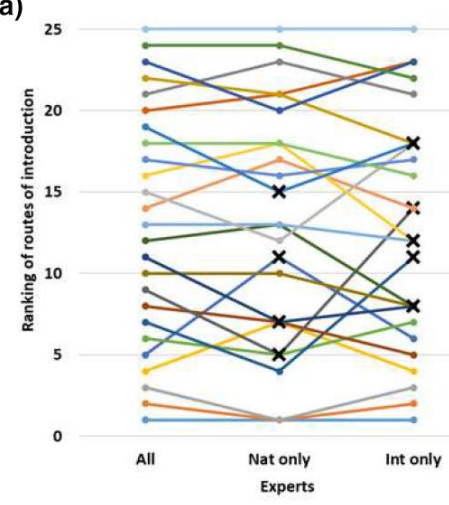

(c)

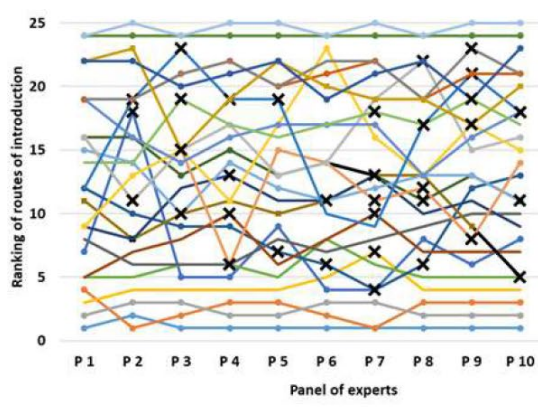

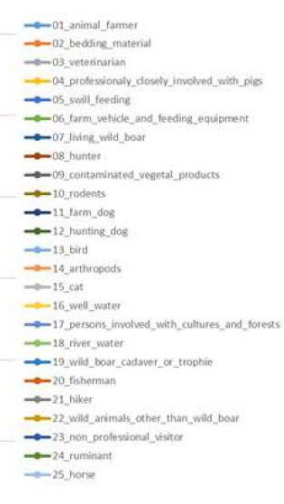

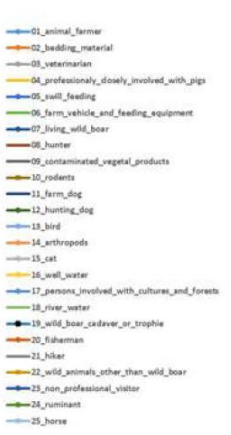

(b) 25

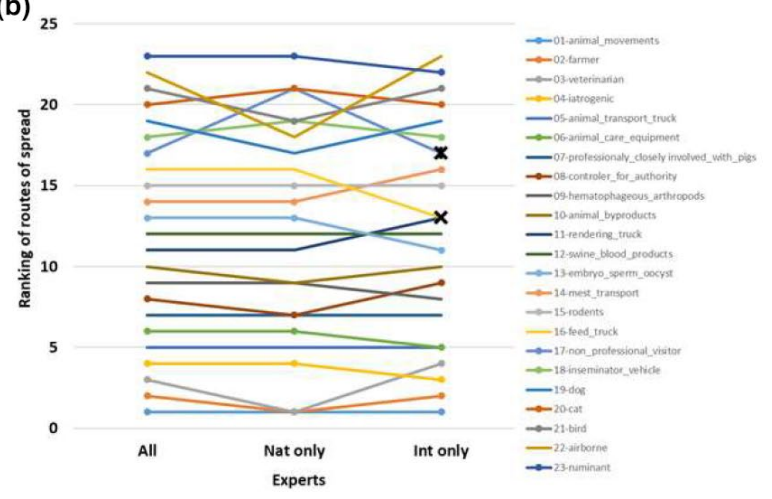

(d)

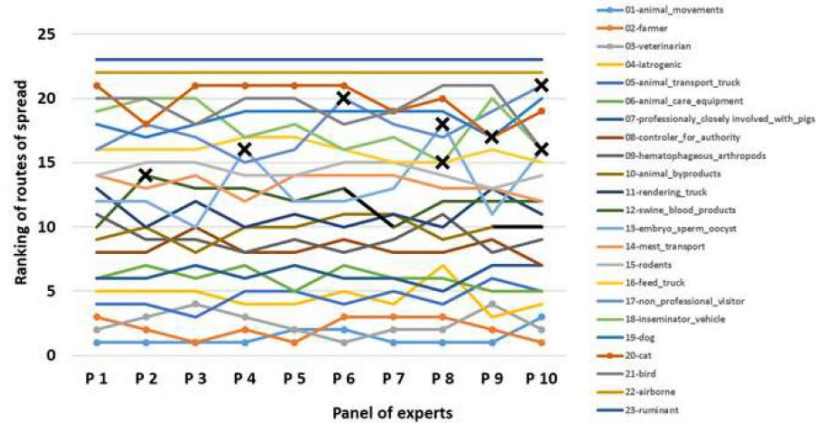

FIGURE 4 Modification in the rank of ASF transmission routes via sensitivity analysis on the origin of experts and on the expert panel, both for introduction [a] and [c] and spread [b] and [d], respectively. All: expert from national and international origin; Nat only: only expert from national origin; Int only: only experts from international origin; P: ten random panels with different composition of field expertise. X: more than three ranks of difference in the ranking between the particular situation and the situation with all experts

reflects that, in the Belgian epidemiological context (i.e. a few holdings with outdoor access, professionalized operators, a very strict regulatory framework, a strong regionalization of pig and wild boar population densities), indirect transmission through (human-related) mechanical vectors of the virus can play a higher role than direct contacts with infected wild boar. This highlights the role of the so-called 'human factor' in the epidemiology of the disease (Zani et al., 2019). However, direct contacts must be especially considered in case of outdoor access. In the Belgian context, very few pig holdings were still currently active within the regulated zones in Belgium and they were all closed. Bedding material as well as vegetal products used for feed may constitute one of the most controversial routes, especially since national and international experts had diverging opinion on these factors.

Scientific data on the persistence of the virus on vegetal products in field conditions and on representative matrices are scarce in comparison with the putative frequency of contacts pigs can have with (i.e. when used as feed) and the efficiency of these routes (Niederwerder et al., 2019; Stoian et al., 2020). These materials deserve attention and more representative experimental studies, especially for feed, are needed. Fischer and collaborators recently showed that the risk of ASFV transmission via contaminated crops is most likely low if they are incubated for at least $2 \mathrm{hr}$ at room temperature (Fischer et al., 2020). Nonetheless, the EU commission recommends the storage for 1 month of any grains or grass used for feed and the ban of straw used for bedding for 3 months (unless these are specifically treated to inactivate ASF virus) to minimize the risk of transmission as much as possible. In contrast, even if bedding material was already suspected to be involved in ASF transmission in some European countries (Nurmoja et al., 2018; Olš̌evskis et al., 2016), this was less expected to play an important role as the use of straw as bedding material is a rare practice in most of the (closed) commercial pig holdings.

The high rank attributed to swill feeding can be surprising in the Belgian context, as this practice is forbidden by regulation since the previous incursion of African swine fever in Belgium in 1985 was due to this transmission route (pigs were fed with contaminated pig meat from the Iberian peninsula where the virus was circulating; Biront et al, 1987). Swill feeding is also forbidden by European regulation (Regulation (EC) n¹069/2009 of the European Parliament and the Council of 21 October 2009 laying down health rules regarding animal by-products and derived products not intended for human consumption). This route is known to be very efficient for ASF transmission between domestic pigs given the resistance of the virus, particularly in meat. On one hand, experts scored very high both the likelihood of contamination (median score $=4$, medium uncertainty) and the transmission (median score $=5$, medium uncertainty) of swill feeding if it was containing contaminated meat. On the other hand, the frequency of contact with pigs was rated much lower (median score $=1$, low 
uncertainty) due to regulation constraints. However, the relative importance of the risk associated with swill feeding, for example fraudulent consignments of contaminated meat from other contaminated areas, poaching of potentially infected Belgian wild boar should be reminded to all operators, especially hobbyists and petting zoos or those with less experience in the pig sector. It must be highlighted that ranking for swill feeding diverged between Belgian (national) and non-Belgian (international) experts (Data S6). The fact that swill feeding obtained the highest ranking according to international experts can probably be explained by the fact that swill feeding has long been shown as a common and historically important transmission route for ASF and forgetting the specific structure of the pig industry in Belgium for which 'backyard' holdings are rare (as usually this factor is more frequently associated with swill feeding practice; (Boklund et al., 2020)).

The risk of ASF introduction via leisure activities or forest work was low ranked by experts. While these activities might not have a significant impact on the risk of ASF introduction into pig holdings, they could be important to affect ASF spread in wildlife (Petit et al., 2019). It should be noted that most of the low ranked transmission routes were associated with a high uncertainty and also deserve more studies, especially for a putative role of insects in transmission cycles in European conditions (Bonnet et al., 2020; de Carvalho Ferreira et al., 2014; Olesen, Hansen et al., 2018; Olesen, Lohse et al., 2018; Saegerman et al., 2020) (EFSA, 2010).

At the pig holdings level, the semi-quantitative assessment highlighted three groups in the transmission routes between holdings. The most important routes were animal movements (for which it should be reminded that the experts were clearly requested to assess the transmission routes before any management measures in place, that is before any detection of an index case and putative first propagation events), the operator himself (the farmer), the practitioner (veterinarian), the iatrogenic route, animal transport trucks and animal care equipment. Moreover, the assessment of these six routes was associated with a lower uncertainty compared with the assessment of most of the other routes, which further reinforces their relative importance. Animal movement networks are essential in understanding and containing the spread of infectious diseases in farming industries. Pig movement networks have been analysed and found to be useful in predicting the risk of infectious disease outbreaks (Halasa et al., 2016; Lentz et al., 2016). Similar models are useful and urgently needed for every country threatened by ASF introduction to their pig industry in order to focus on early measures on high-risk holdings ('super spreaders'). It should be stressed that the farmer and the veterinarian(s) on the farm are again identified as very important transmission routes (mechanical vectors) for ASF spread between holdings. This underlines their roles and responsibilities in ensuring the implementation of biosecurity measures (Beltrán-Alcrudo et al., 2017).

The effect of live animal movements on disease spread is dependent on the course of the disease. For a disease such as ASF with a short incubation period and quite pathognomonic clinical signs, the spread through animal movements before the first detection of the infection is expected to be minimal. During the period, ASF was present in the wild reservoir in Belgium additional vigilance was introduced through the legal requirements to report any clinical signs or abnormal mortality in pigs (with routine sampling and laboratory analysis for ASF). This was aimed at detecting a possible index case in the domestic population as early as possible. Moreover also the biosecurity (especially external biosecurity) was increased. The combination of these measures aimed at limiting possible spread of the virus once it was introduced into the domestic population. However, the experience of the previous ASF epidemic in Belgium must remind that late reporting can seriously jeopardize animal health and the economy of the country (Biront et al., 1987). In some cases (e.g. large holding with separated operating units), this risk has been associated with a relatively low apparent mortality rate (due to a low inter-unit spreading rate of the virus, that is the virus spreading fast between animals within a closed unit but slower between units). This scenario has been observed during some ASF outbreak in Eastern European domestic pig holdings (Schulz et al., 2017). These factors can therefore delay the detection of virus circulation in the index holding if samples are not sent early for analysis in case of clinical syndrome and facilitates an initial spread via animal movements.

The limitations of this study are those related to every expert elicitations, in particular those carried out by mail during which further explanation cannot be obtained, as well as to qualitative assessment. Furthermore, ASF epidemiology is complex, especially when dealing with the interface of the domestic pig sector and wildlife. Finding experts who cover every field of expertise (virology, epidemiology, pig sector, wildlife) is difficult. However the response rate, the diversity in the collected expertise and the performed sensitivity analyses on the results allow to give robust confidence in the final results. Interestingly, sensitivity analysis highlights the importance to consider all criteria (likelihood of contamination by ASF, likelihood of transmission of ASF to domestic pigs, and frequency of contact between domestic pigs) in ASF transmission routes and to use a sufficient number of experts coming from different fields of expertise in order to produce robust scientific advice for decision-making. Many uncertainties remain or data need further validation (e.g. the role of feed or haematophagous arthropods). This study especially associated a level of uncertainty on each assessment made.

Results of this study demonstrate the importance of indirect routes, especially the human-related ones, for ASF introduction into a pig holding considering the Belgian context for ASF. Considering further ASF spread between pig holdings, animal movements and once again, humans as passive vectors were shown to be the most important transmission routes. Increasing pig owner awareness about biosecurity, in particular about external biosecurity, and key features of the disease, as well as performing regular risk-based surveillance by the animal health authorities in pig holdings helped to tackle the risk associated to ASF for the Belgian pig industry. As the structure of the pig sector of most of the Western European countries is similar to that of Belgium (few non-commercial farms and relatively high biosecurity level), conclusions of this study could be extrapolated to them. 


\section{ACKNOWLEDGEMENTS}

The authors deeply acknowledge all the national and international experts who participated to this survey (exhaustive list in Data S2). They are also grateful to the members of the Scientific Committee established at the Belgian Federal Agency for the Safety of the Food Chain for their advices concerning the analysis and the interpretation of the results. The authors acknowledge V. Clavier, M. Logeot, $X$. Patigny and A. E. Popa who participated in the activities of the working group for the preparation of the related opinion of the Scientific Committee established at the FASFC as observers of the FASFC; F. De Meersman, Y. Dejaegher, G. Fichefet, E. Goosens, V. Mohy, A. Noiret, G. Schmitz, L. Pluym, R. Reyntiens (stakeholders); M. Herman, M.-J. Goffaux, A. Licoppe, J.-P. Scohy, D. Arnould, A Lnden, J. Hooyberghs, P. Houdart and J.-F. Heymans as well as the other members from the strategic Committee for the management of the ASF outbreak in wildlife in Belgium (risk managers) who provided important information on the epidemiological situation.

\section{CONFLICT OF INTEREST}

The authors declare that there is no conflict of interest.

\section{ORCID}

Axel Mauroy iD https://orcid.org/0000-0002-5101-810X Claude Saegerman iD https://orcid.org/0000-0001-9087-7436 Dominiek Maes iD https://orcid.org/0000-0003-1149-9348 Kevin Morelle iD https://orcid.org/0000-0001-9041-6541 Etienne Thiry (iD https://orcid.org/0000-0002-9585-7933 Jeroen Dewulf (iD https://orcid.org/0000-0001-9843-5990

\section{REFERENCES}

Alonso, C., Borca, M., Dixon, L., Revilla, Y., Rodriguez, F., \& Escribano, J. M. (2018). ICTV virus taxonomy profile: Asfarviridae. Journal of General Virology, 99, 613-614. https://doi.org/10.1099/jgv.0.001049

Bellini, S., Casadei, G., De Lorenzi, G., \& Tamba, M. (2021). A review of risk factors of African swine fever incursion in pig farming within the European Union Scenario. Pathogens, 10, 84. https://doi. org/10.3390/pathogens10010084

Beltrán-Alcrudo, D., Arias, M., Gallardo, C., Kramer, S., \& Penrith, M. L. (2017). African swine fever: Detection and diagnosis - A manual for veterinarians (p. 88). FAO Animal Production and Health Manual No. 19. Rome: Food and Agriculture Organization of the United Nations (FAO).

Biront, P., Castryck, F., \& Leunen, J. (1987). An epizootic of African swine fever in Belgium and its eradication. Veterinary Records, 120, 432-4. https://doi.org/10.1136/vr.120.18.432

Blome, S., Franzke, K., \& Beer, M. (2020). African swine fever - A review of current knowledge. Virus Research, 287, 198099. https://doi. org/10.1016/j.virusres.2020.198099

Boklund, A., Dhollander, S., Chesnoiu Vasile, T., Abrahantes, J. C., Bøtner, A., Gogin, A., ... Mortensen, S. (2020). Risk factors for African swine fever incursion in Romanian domestic farms during 2019. Scientific Reports, 10, 1-13. https://doi.org/10.1038/s41598-020-66381-3

Bonnet, S. I., Bouhsira, E., De Regge, N., Fite, J., Etoré, F., Garigliany, M. M., ... Vial, L. (2020). Putative role of arthropod vectors in African swine fever virus transmission in relation to their bio-ecological properties. Viruses, 12(7), 778. https://doi.org/10.3390/v12070778

Chenais, E., Ståhl, K., Guberti, V., \& Depner, K. (2018). Identification of wild boar-habitat epidemiologic cycle in African swine fever epizootic. Emerging Infectious Diseases, 24, 810-812. https://doi. org/10.3201/eid2404.172127

Cho, K.-H., Kim, H.-J., Kim, Y.-J., Kang, H.-E., Martínez-López, B., \& Lee, J.-B. (2020). Quantitative risk assessment of the African swine fever introduction into the Republic of Korea via legal import of live pigs and pig products. Transboundary and Emerging Diseases, https://doi. org/10.1111/tbed.13689

Costard, S., Jones, B. A., Martínez-López, B., Mur, L., de la Torre, A., Martínez, M., ... Wieland, B. (2013). Introduction of African swine fever into the European Union through Illegal Importation of Pork and Pork Products. PLoS One, 8, e61104. https://doi.org/10.1371/ journal.pone.0061104

Costard, S., Mur, L., Lubroth, J., Sanchez-Vizcaino, J. M., \& Pfeiffer, D. U. (2013). Epidemiology of African swine fever virus. Virus Research, 173, 191-197. https://doi.org/10.1016/j.virusres.2012.10.030

de Carvalho Ferreira, H. C., Tudela Zúquete, S., Wijnveld, M., Weesendorp, E., Jongejan, F., Stegeman, A., \& Loeffen, W. L. A. (2014). No evidence of African swine fever virus replication in hard ticks. Ticks and Tick-borne Diseases, 5, 582-589. https://doi.org/10.1016/j. ttbdis.2013.12.012

Dufour, B., Plée, L., Moutou, F., Boisseleau, D., Chartier, C., Durand, B., Ganière, J. P., Guillotin, J., Lancelot, R., Saegerman, C., Thébault, A., Hattenberger, A. M., \& Toma, B. (2011). A qualitative risk assessment methodology for scientific expert panels. Revue Scientifique et Technique de l'Office International des Epizooties, 30(3), 673-681. https://doi.org/10.20506/rst.30.3.2063

EFSA. (2020). Scientific report on the epidemiological analyses of African swine fever in the European Union. EFSA Journal, 18, 5996. https:// doi.org/10.2903/j.efsa.2020.5996

EFSA Panel on Animal Health and Welfare. (2010). Scientific Opinion on the role of tick vectors in the epidemiology of Crimean Congo hemorrhagic fever and African swine fever in Eurasia. EFSA Journal, 8, 1-156. https://doi.org/10.2903/j.efsa.2010.1703

EFSA Panel on Animal Health and Welfare. (2014). Scientific opinion on African swine fever. EFSA Journal, 12(3628), 77. https://doi. org/10.2903/j.efsa.2014.3628

Fila, M., \& Woźniakowski, G. (2020). African swine fever virus - the possible role of flies and other insects in virus transmission. Journal of Veterinary Research, 1-7. https://doi.org/10.2478/jvetres-2020-0001

Fischer, M., Mohnke, M., Probst, C., Pikalo, J., Conraths, F. J., Beer, M., \& Blome, S. (2020). Stability of African swine fever virus on heattreated field crops. Transboundary and Emerging Diseases, 67(6), 2318-2323. https://doi.org/10.1111/tbed.13650

Garigliany, M., Desmecht, D., Tignon, M., Cassart, D., Lesenfant, C., Paternostre, J., ... Linden, A. (2019). Phylogeographic analysis of African swine fever virus, Western Europe, 2018. Emerging Infectious Diseases, 25(1), 184-186. https://doi.org/10.3201/eid2501.181535

Gilliaux, G., Garigliany, M., Licoppe, A., Paternostre, J., Lesenfants, C., Linden, A., \& Desmecht, D. (2019). Newly emerged African swine fever virus strain Belgium/Etalle/wb/2018: Complete genomic sequence and comparative analysis with reference p72 genotype II strains. Transboundary and Emerging Diseases, 66, 2566-2591. https://doi.org/10.1111/tbed.13302

Guinat, C., Gogin, A., Blome, S., Keil, G., Pollin, R., Pfeiffer, D. U., \& Dixon, L. (2016). (12. March): Transmission routes of African swine fever virus to domestic pigs: Current knowledge and future research directions. The Veterinary Record, 178, 262-267. https://doi.org/10.1136/ vr.103593

Halasa, T., Bøtner, A., Mortensen, S., Christensen, H., Toft, N., \& Boklund, A. (2016). Simulating the epidemiological and economic effects of an African swine fever epidemic in industrialized swine populations. Veterinary Microbiology, 193, 7-16. https://doi.org/10.1016/j. vetmic.2016.08.004

Herrera-Ibatá, D. M., Martínez-López, B., Quijada, D., Burton, K., \& Mur, L. (2017). Quantitative approach for the risk assessment of African 
swine fever and Classical swine fever introduction into the United States through legal imports of pigs and swine products. PLoS One, 12, e0182850. https://doi.org/10.1371/journal.pone.0182850

Ito, S., Jurado, C., Sánchez-Vizcaíno, J. M., \& Isoda, N. (2020). Quantitative risk assessment of African swine fever virus introduction to Japan via pork products brought in air passengers' luggage. Transboundary and Emerging Diseases, 67, 894-905. https://doi.org/10.1111/tbed.13414

Jurado, C., Martínez-Avilés, M., De La Torre, A., Štukelj, M., de Carvalho Ferreira, H. C., Cerioli, M., Sánchez-Vizcaíno, J. M., \& Bellini, S. (2018). Relevant measures to prevent the spread of African swine fever in the European Union domestic pig sector. Frontiers in Veterinary Science, 5, 77. https://doi.org/10.3389/fvets.2018.00077

Lentz, H. H. K., Koher, A., Hövel, P., Gethmann, J., Sauter-Louis, C., Selhorst, T., \& Conraths, F. J. (2016). Disease spread through animal movements: A static and temporal network analysis of pig trade in Germany. PLoS One, 11, e0155196. https://doi.org/10.1371/journ al.pone.0155196

Linden, A., Licoppe, A., Volpe, R., Paternostre, J., Lesenfants, C., Cassart, D., ... Cay, A. B. (2019). Summer 2018: African swine fever virus hits north-western Europe. Transboundary and Emerging Diseases, 66, 54 55. https://doi.org/10.1111/tbed.13047

Mur, L., Martínez-López, B., Martínez-Avilés, M., Costard, S., Wieland, B., Pfeiffer, D. U., \& Sánchez-Vizcaíno, J. M. (2012). Quantitative risk assessment for the introduction of African swine fever virus into the European Union by legal import of live pigs. Transboundary and Emerging Diseases, 59, 134-144. https://doi. org/10.1111/j.1865-1682.2011.01253.x

Niederwerder, M. C., Stoian, A. M. M., Rowland, R. R. R., Dritz, S. S., Petrovan, V., Constance, L. A., ... Hefley, T. J. (2019). Infectious dose of African swine fever virus when consumed naturally in liquid or feed. Emerging Infectious Diseases, 25, 891-897. https://doi. org/10.3201/eid2505.181495

Nurmoja, I., Mõtus, K., Kristian, M., Niine, T., Schulz, K., Depner, K., \& Viltrop, A. (2018). Epidemiological analysis of the 2015-2017 African swine fever outbreaks in Estonia. Preventive Veterinary Medicine, 2018, 2015-2017. https://doi.org/10.1016/j.prevetmed.2018.10.001

OIE. (2019). Terrestrial animal Health Code sanitaire. Chapter 15. Infection with African swine fever virus. https://www.oie.int/filea dmin/Home/fr/Health_standards/tahc/current/chapitre_asf.pdf

OIE. (2020). WAHIS, Immediate notification for the first occurrence of a listed disease (African Swine Fever in wildboar) in the country. https://www.oie.int/wahis_2/public/wahid.php/Reviewreport/ Review?reportid $=35705$

Olesen, A. S., Belsham, G. J., Rasmussen, T. B., Lohse, L., Bødker, R., Halasa, T., Boklund, A., \& Bøtner, A. (2020). Potential routes for indirect transmission of African swine fever virus into domestic pig herds. Transboundary and Emerging Diseases, 67(4), 1472-1484. https://doi.org/10.1111/tbed.13538

Olesen, A. S., Hansen, M. F., Rasmussen, T. B., Belsham, G. J., Bødker, R., \& Bøtner, A. (2018). Survival and localization of African swine fever virus in stable flies (Stomoxys calcitrans) after feeding on viremic blood using a membrane feeder. Veterinary Microbiology, 222, 25-29. https://doi.org/10.1016/j.vetmic.2018.06.010

Olesen, A. S., Lohse, L., Hansen, M. F., Boklund, A., Halasa, T., Belsham, G. J., ... Bødker, R. (2018). Infection of pigs with African swine fever virus via ingestion of stable flies (Stomoxys calcitrans). Transboundary and Emerging Diseases, 65, 1152-1157. https://doi.org/10.1111/ tbed.12918

Oḷ̌̌evskis, E., Guberti, V., Seržants, M., Westergaard, J., Gallardo, C., Rodze, I., \& Depner, K. (2016). African swine fever virus introduction into the EU in 2014: Experience of Latvia. Research in Veterinary Science, 105, 28-30. https://doi.org/10.1016/j.rvsc.2016.01.006

Petit, K., Dunoyer, C., Fischer, C., Hars, J., Baubet, E., López-Olvera, J. R., ... Saegerman, C. (2019). Assessment of the impact of forestry and leisure activities on wild boar spatial disturbance with a potential application to ASF risk of spread. Transboundary and Emerging Diseases, 67(3), 1164-1176. https://doi.org/10.1111/tbed.13447

Roelandt, S., Van der Stede, Y., D'hondt, B., \& Koenen, F. (2017). The assessment of African Swine fever virus risk to Belgium Early 2014, using the Quick and Semiquantitative Pandora Screening Protocol. Transboundary and Emerging Diseases, 64, 237-249. https://doi. org/10.1111/tbed.12365

Rowlands, R. J., Michaud, V., Heath, L., Hutchings, G., Oura, C., Vosloo, W., ... Dixon, L. K. (2008). African swine fever virus isolate, Georgia, 2007. Emerging Infectious Diseases, 14, 1870-1874. https://doi. org/10.3201/eid1412.080591

Saegerman, C. (2018). Découverte inattendue de la peste porcine en Belgique. Epidémiol et Santé Anim, 73, 147-164. https://orbi. uliege.be/bitstream/2268/232950/1/AEEMA_2018-73_17_Saege rman-V2.pdf

Saegerman, C., Bonnet, S., Bouhsira, E., De Regge, N., Fite, J., Etoré, F., ... Vial, L. (2020). An expert opinion assessment of blood-feeding arthropods based on their capacity to transmit African swine fever virus in Metropolitan France. Transboundary and Emerging Diseases, https://doi.org/10.1111/tbed.13769

Sánchez-Vizcaíno, J. M., Mur, L., Gomez-Villamandos, J. C., \& Carrasco, L. (2015). An update on the epidemiology and pathology of African swine fever. Journal of Comparative Pathology, 152, 9-21. https://doi. org/10.1016/j.jcpa.2014.09.003

Sánchez-Vizcaíno, J. M., Mur, L., \& Martínez-López, B. (2012). African Swine fever: an epidemiological update. Transboundary and Emerging Diseases, 59, 27-35. https://doi. org/10.1111/j.1865-1682.2011.01293.x

Sauter-Louis, C., Forth, J. H., Probst, C., Staubach, C., Hlinak, A., Rudovsky, A., ... Bock, S. (2020). Joining the club: First detection of African swine fever in wild boar in Germany. Transboundary and Emerging Diseases. https://doi.org/10.1111/tbed.13890

Schulz, K., Staubach, C., \& Blome, S. (2017). African and classical swine fever: Similarities, differences and epidemiological consequences. Veterinary Research, 48, 84. https://doi.org/10.1186/s1356 7-017-0490-x

SciCom. (2020). Opinion 06-2020 of the Scientific Committee established at the Federal Agency for the Safety of the Food Chain of 24/03/2020.Semi-quantitative risk assessment of potential routes of introduction of African Swine Fever from wildlife to domestic pig holdings and subsequent spread (dossier SciCom 2019/11 - selftasking mandate). Retrieved from http://www.afsca.be/scientific committee/opinions/2020/_documents/Opinion06-2020_SciCo m2019-11_ASF.pdf

Stoian, A. M. M., Petrovan, V., Constance, L. A., Olcha, M., Dee, S., Diel, D. G., ... Niederwerder, M. C. (2020). Stability of classical swine fever virus and pseudorabies virus in animal feed ingredients exposed to transpacific shipping conditions. Transboundary and Emerging Diseases, 67(4), 1623-1632. https://doi.org/10.1111/ tbed.13498

Sugiura, K., \& Haga, T. (2018). A rapid risk assessment of african swine fever introduction and spread in Japan based on expert opinions. Journal of Veterinary Medical Science, 80(11), 1743-1746. https://doi. org/10.1292/jvms.18-0543

Vergne, T., Andraud, M., Bonnet, S., De Regge, N., Desquesnes, M., Fite, J., ... Bouhsira, E. (2020). Mechanical transmission of African swine fever virus by Stomoxys calcitrans: Insights from a mechanistic model. Transboundary and Emerging Diseases. https://doi. org/10.1111/tbed.13824

Vergne, T., Gogin, A., \& Pfeiffer, D. U. (2017). Statistical exploration of local transmission routes for African swine fever in pigs in the Russian Federation, 2007-2014. Transboundary and Emerging Diseases, 64, 504-512. https://doi.org/10.1111/tbed.12391 
Zani, L., Dietze, K., Dimova, Z., Forth, J. H., Denev, D., Depner, K., \& Alexandrov, T. (2019). African swine fever in a Bulgarian backyard farm-a case report. Veterinary Sciences, 6, 94. https://doi. org/10.3390/vetsci6040094

\section{SUPPORTING INFORMATION}

Additional supporting information may be found online in the Supporting Information section.
How to cite this article: Mauroy A, Depoorter P, Saegerman

$C$, et al. Semi-quantitative risk assessment by expert elicitation of potential introduction routes of African swine fever from wild reservoir to domestic pig industry and subsequent spread during the Belgian outbreak (2018-2019).

Transbound Emerg Dis. 2021;00:1-13. https://doi. org/10.1111/tbed.14067 